\title{
LA RAPPRESENTAZIONE DEL MARE NELL'ARTE FRANCESCANA
}

\section{HISASHI YAKOU}

UDC: 75.052 .046 .3

Original scientific paper

Manuscript received: 18. 02. 2016.

Revised manuscript accepted: 21. 04. 2016.

DOI: 10.1484/J.HAM.5.111353
H. Yakou

Hokkaido University

Department of Theory and History of Arts, Graduate School of Letters Kita 10, Nishi 7, Sapporo

It is well known that St. Francis of Assisi was eager to go overseas and evangelize the pagans. After the saint's death, his companion friars went as far as India and China. However, we find only passing references to the sea or voyages by sea in the Franciscan literature. Moreover, in the field of figurative art, even if occasionally we see scenes of shipwrecks, succored by the intercession of St. Francis or a Franciscan friar, these just seem to follow a precedent scheme of traditional Christian iconographies such as Calling of Peter, etc. and do not reflect a new dimension of experience accumulated by Franciscan missionaries. In this paper, a fragmentary fresco conserved in the diocesan museum of Fidenza, representing a shipwreck scene with a Franciscan friar, is examined to identify the episode. Then, Christian tombstones excavated in Quanzhou and Yangzhou, China, are surveyed to measure the extent to which the reciprocal influences between West and East, in terms of style and iconography, were significant for the artifacts of the Late Middle Ages of Italy.

Keywords: Fidenza, Thane, Quanzhou, Yangzhou, Franciscan mission, martyrdom

\section{LA SCENA DI NAUFRAGIO CON UN FRATE FRANCESCANO A FIDENZA}

Nel museo diocesano di Fidenza è esposto un frammento di affresco staccato dalla sua posizione originale, un locale adiacente la cripta del Duomo ${ }^{1}$ (figg. 1, 2). Fidenza è la città emiliana che vanta una cattedrale abbellita dalle sculture ed i rilievi prodotti dalla bottega di Antelami, ma i dipinti medievali ivi conservati sono stati, fino ad oggi, solo sporadicamente menzionati dalla critica ${ }^{2}$. L'affresco rappresenta due santi, Santa Caterina d'Alessandria e San Bartolomeo, inquadrati nelle cornici dipinte, e una scena di naufragio in cui si vedono le vele e gli alberi della nave spezzati. Sulla barca ci sono due persone rivolte a sinistra, tra cui almeno una alza le mani congiunte in segno di supplica o di ringraziamento, e un'altra persona che sembra già morta. Inoltre, un frate francescano con barba e baffi, anche lui rivolto a sinistra, porge libri (forse due volumi) ${ }^{3}$. La scena è chiusa a destra da un corpo gigantesco (di San Cristoforo?), di cui rimane solo il drappeggio della parte femorale. La data del dipinto non è certa, ma collocabile nei primi decenni del Trecento ${ }^{4}$.
È stata fatta l'ipotesi molto audace che si tratti di una scena di naufragio sul fiume Po, dove i pellegrini sono scampati a un uragano per un miracolo di San Francesco d'Assisi'5. Nessuna fonte menziona questo episodio, ma il riquadro votivo potrebbe essere legato a un luogo, cioè il duomo di Fidenza, dove si esprime una devozione particolare a San Francesco, come testimonia la rappresentazione insolita di questo santo sulla parete absidale ${ }^{6}$.

L'episodio delle tenebre dissipate e il beneficio della luce dato da Dio mentre San Francesco e un suo compagno viaggiavano lungo il Po nella zona paludosa tra la Lombardia e la Marca Trevigiana è raccontato solo da Bonaventura nella Legenda Maior, V, 12 e nella Legenda Minor, V, 8. È l'unico brano di tutte le leggende francescane in cui appaia il nome del Po. Il paragrafo precedente della Legenda Minor, V, 7, tratta del miracolo di scampato naufragio e moltiplicazione del pane avvenuto sulla rotta tra la Schiavonia (Dalmazia) e Ancona. Francesco era al rientro dal fallito tentativo di viaggio in Siria7. Se è vero che la scena del frammento fidentino è ambientata sul Po, l'ideatore del dipinto avrebbe osato congiungere due episodi, inclusi uno dopo l'altro nella

${ }^{1}$ G. GREGORI (a cura di), Il Museo del Duomo. Il Museo Diocesano di Fidenza, Parma, 2003, pp. 85-86.

${ }^{2}$ Per l'architettura e la scultura del Duomo di Fidenza, rimando a Y. KOJIMA, Storia di una cattedrale - il Duomo di San Donnino a Fidenza: il cantiere medievale, le trasformazioni, i restauri, Pisa, 2006. Un prezioso riferimento al Giudizio universale, raffigurato sulla parete absidale del Duomo fidentino, si trova in W. R. COOK, Images of St Francis of Assisi in painting, stone and glass from the earliest images to ca. 1320 in Italy, Firenze, 1999, p. 94, ma con una data al 1270-80. A mio parere, il Giudizio è stato dipinto prima del 1268, l'anno in cui la città di Fidenza, allora Borgo San Donnino, è stata profondamente danneggiata dai parmigiani, tanto che le fonti parlano di distruzione, perché non riprendessero i lavori alla Cattedrale.

${ }_{3}$ Questa figura viene identificata come San Nicola di Bari da G. (M.) PONZI, "Frammenti di affreschi del Duomo di Fidenza," Il Risveglio - il settimanale della città e della diocesi di Fidenza, 07/12/2012 (reperibile a http://lucecolore.blogspot.jp/2012/12/frammenti-di-affreschi-del-duomo-di.html).

4 Poiché la ricostruzione della città di Fidenza è stata consentita da Parma solo nel 1302, l'affresco non può risalire a prima di quell'anno. Kojima, op. cit. (n. 2), p. 20. La Santa Caterina di Fidenza somiglia vagamente alla figura della stessa santa del Battistero di Parma. Quest'ultima, di qualità più alta, è stata attribuita a Buffalmacco e datata tra il 1327 ca e il 1336 da L. BELLOSI, Buffalmacco e il Trionfo della Morte, Milano, 2003 (2 ed.), p. 72 et passim, fig. 136. Penso che questa possa essere la data di riferimento anche dell'affresco di Fidenza.

${ }^{5}$ A. AIMI, Storia di Fidenza, Parma, 2003, p. 83. San Francesco, di passaggio a Fidenza nel 1215, avrebbe moltiplicato il pane per una cena. Cfr. Ibidem, p. 55. ${ }^{6}$ Ibidem, p. 72; H. YAKOU, “The Last Judgment in the Cathedral of Fidenza and the Eschatological Images in the Franciscan Context," in: B. R. FRANCO, B. A. MULVANEY (a cura di), The World of St. Francis of Assisi, atti del convegno, Siena, 2016 (in preparazione).

${ }_{7}$ Nella Legenda Maior, lo stesso evento appare nel cap. IX, 5, che non ha alcun collegamento con il racconto del Po. 


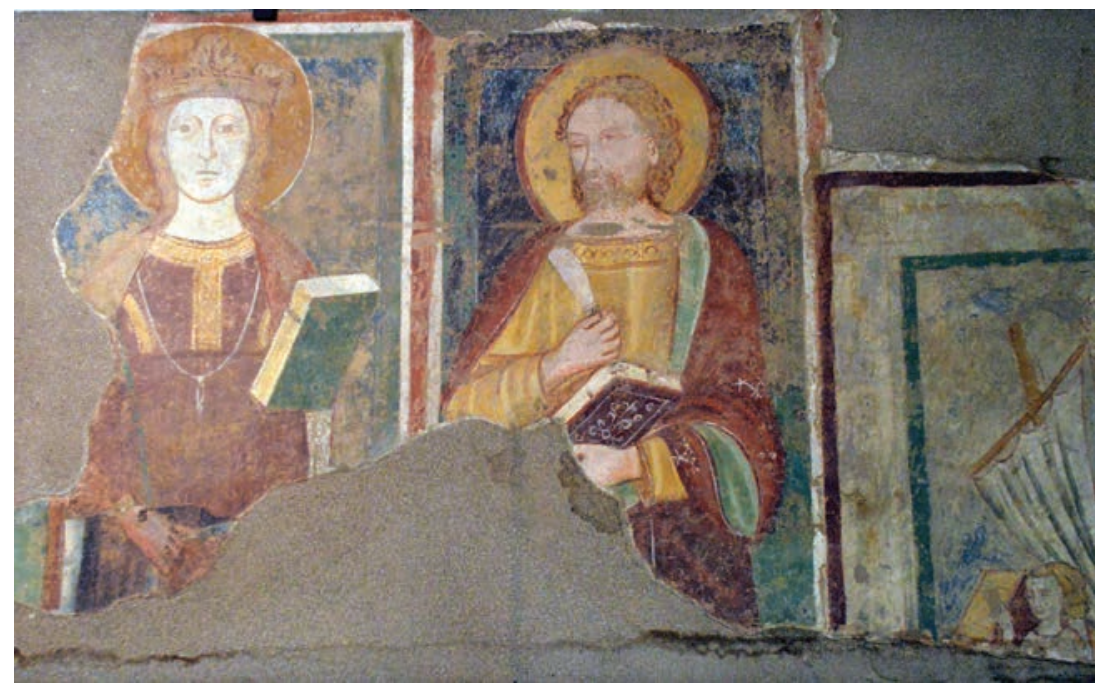

Fig. 1. Pittore emiliano, Santa Caterina d'Alessandria e San Bartolomeo, Fidenza, Museo diocesano.

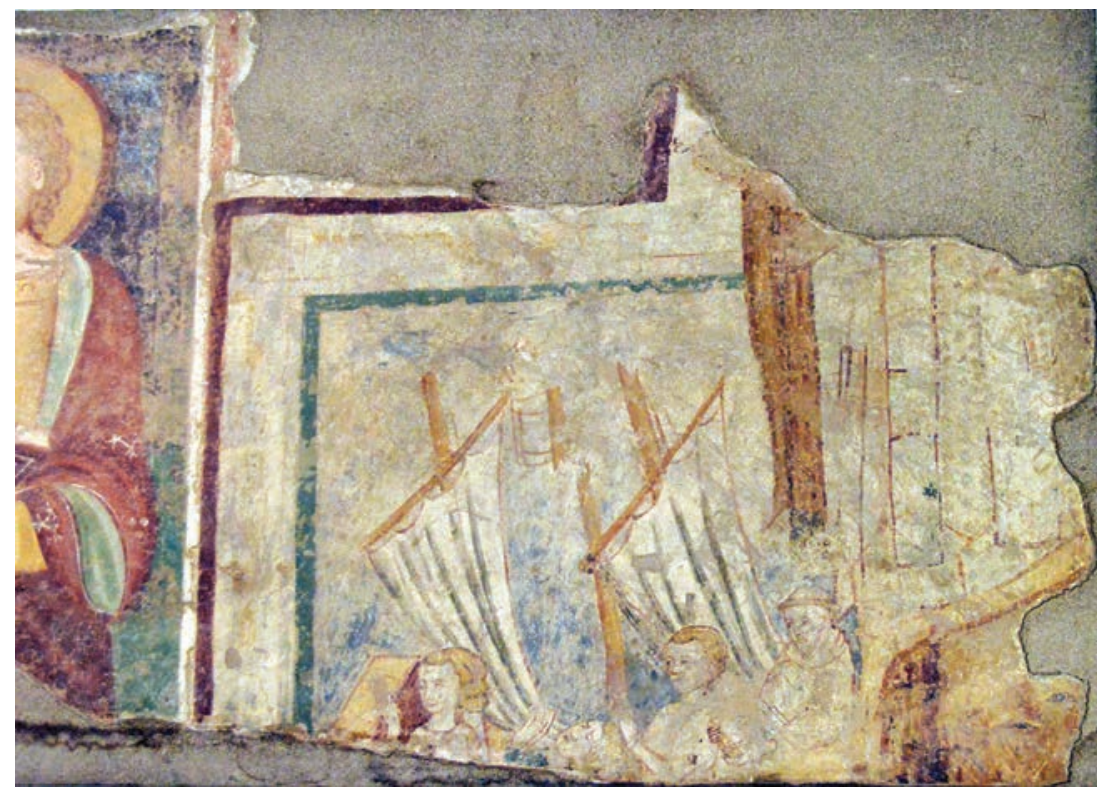

Fig. 2. Pittore emiliano, Scena di naufragio con un frate francescano, Fidenza, Museo diocesano.

Legenda Minor e tutti e due attinenti all'acqua ${ }^{8}$. Per un ex voto, questa congettura mi pare possibile anche se non è verificabile. L"ipotesi più plausibile è che la scena tratti dello scampato naufragio già citato e raccontato non solo da San Bonaventura, ma anche da Tommaso da Celano', anche se non si può escludere che si riferisca ad un simile episodio successo al committente del dipinto.

La scena del naufragio è rappresentata anche in un riquadro sulla tavola d'altare messa nella cappella Bardi della Chiesa di Santa Croce a Firenze ${ }^{10}$. I marinai invocano
San Francesco con le mani giunte, lo stesso gesto che abbiamo già visto nel frammento di Fidenza. Si è scelta un'ambientazione sul mare con una nave quando il ciclo della vita di San Francesco comprendeva più scene possibili, come nel caso della tavola della Cappella Bardi, che conta ben venti episodi, e delle illustrazioni del Lignum Vitae Sancti Francisci, una delle quali si trova a Padova, nel cosiddetto Parlatorio del Convento di Sant'Antonio ${ }^{\prime \prime}$.

Lì si vede solo la figura di San Francesco seduto in una barca con un grosso libro sulla sua destra. Alessandro Simbeni suggerisce che questa scena, ormai quasi irriconoscibile, dipinta in un tondo di fogliame, potrebbe rappresentare il viaggio del santo in Terrasanta descritta nella Legenda Maior, IX, 5, anche se è posta dopo il funerale di San Francesco, cioè, nella stessa successione della tavola Bardi ${ }^{12}$, e quindi non dovrebbe essere necessariamente interpretata come se narrasse un episodio o un miracolo post mortem.

C’è la possibilità che si tratti di un altro episodio nel golfo di Gaeta, Predica alla folla da una barca, raccontato solo da Bonaventura ma ben due volte, nella Legenda Maior, XII, 6, e nella Legenda Minor, $\mathrm{V}, 4$, come è successo per il prodigio della luce che illumina la via lungo il $\mathrm{Po}^{13}$. Francesco, per fuggire dai fedeli che gli si affollavano intorno e predicare con tranquillità, è salito su una barca che l'ha miracolosamente allontanato dalla riva. Il santo segue così le orme di Cristo, che ha predicato in una barca sul lago di Galilea (Matteo, 13; Luca, 5).

Comunque sia, in tutti questi casi il riferimento al mare è troppo ambiguo, se si considera l'importanza che in quell'epoca aveva non solo per il commercio, ma anche per l'esperienza dei missionari, soprattutto dei frati francescani. E sembra che la composizione ricalchi semplicemente lo schema di episodi più comuni come Il miracolo delle navi cariche di grano, e gli altri miracoli compiuti da San Nicola di Bari, La pesca miracolosa, La chiamata di Pietro e Andrea, etc. Pare che l'espansione delle tratte marittime non abbia dato nessun stimolo ad ampliare l'immaginario geografico, collettivo ad un mondo esteso nello spazio, e creare così una nuova iconografia per gli episodi sul mare.

\section{MISSIONARI FRANCESCANI IN ASIA E L'ARTE FIGURATIVA}

I francescani, essendo entusiasti missionari della fede Cristiana in paesi pagani, attraversavano il mare per raggiun-

\footnotetext{
${ }^{8}$ Una raccolta dei miracoli post mortem di Francesco che salvano i naufraghi sia sul mare che su laghi o fiumi, si trova nel Tractatus de miraculis, par. 8187, di Tommaso da Celano. Tutti gli episodi ivi riportati, tranne uno, sono stati ripresi dalla sezione dei miracoli postumi della Legenda maior, Mir. IV, 1-5 di Bonaventura.

9 Vita prima, par. 55 (pars I, cap. 26). Tommaso da Celano ripete lo stesso episodio nel suo Tractatus de miraculis, par. 33.

${ }^{10}$ Per le vicende critiche della tavola Bardi, prevalentemente attribuita a Coppo di Marcovaldo, rimando alla scheda sintetica di A. Tartuferi per il catalogo della mostra L'arte di Francesco. Capolavori d'arte italiana e terre d'Asia dal XIII al XV secolo, a cura di A. TARTUFERI, F. D'ARELLI, Firenze, 2015, p. 280. Se la tavola è stata eseguita nel 1245-50, il pittore poteva consultare solo la Vita prima di Tommaso da Celano, visto che le altre fonti sono state compilate solo dopo la metà del Duecento. Su questo cfr. C. FRUGONI, Francesco e l'invenzione delle stimmate, Torino, 1993, pp. 379-380.

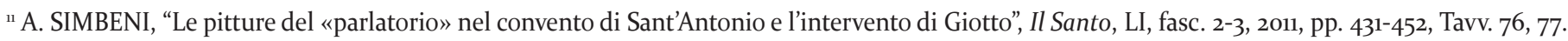

${ }^{12}$ Ibidem, p. 438.

${ }^{13}$ Seguo D. SOLVI, Officina Franciscana, I, Sinossi e indici, Firenze, 2005, pp. 225, 246, per il titolo della scena.
} 


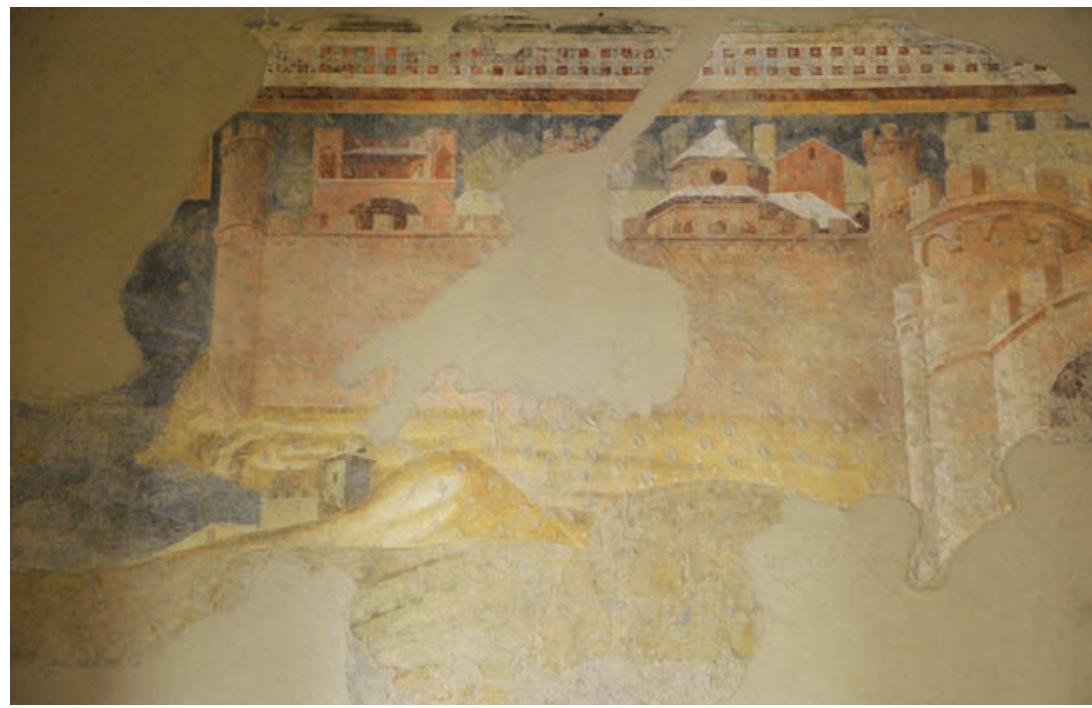

Fig. 3. Ambrogio Lorenzetti, La città di Tana, Siena, Rettorato dell'Università di Siena.

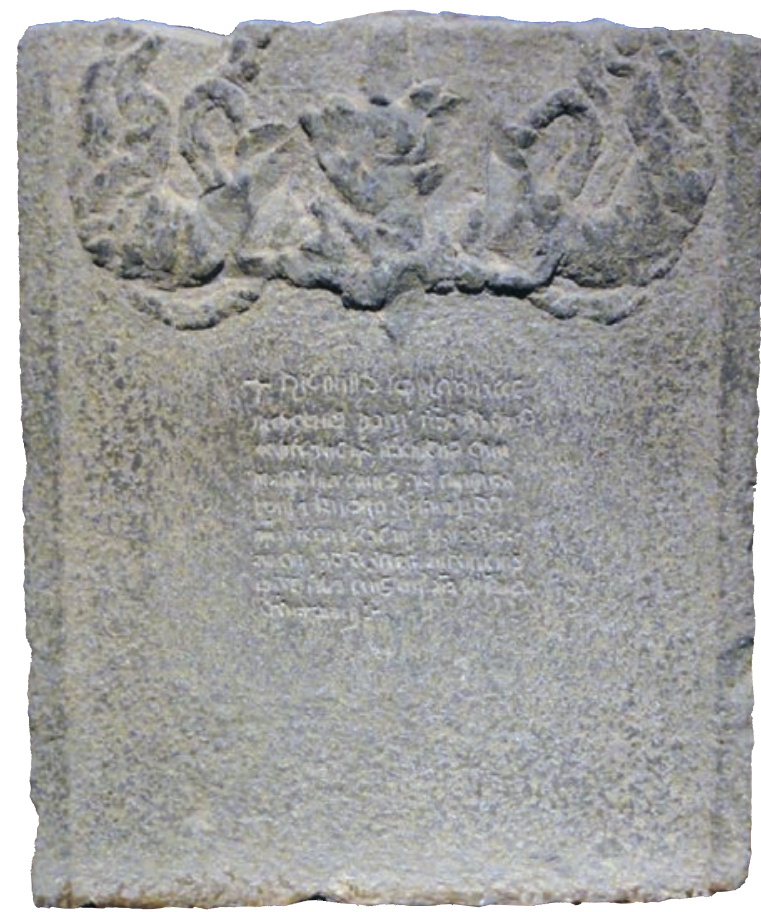

Fig. 4. Lastra tombale di Andrea da Perugia († 1332), Quanzhou, Museo marittimo.

Ora volgiamo il nostro sguardo a Zayton, odierna Quanzhou nella Provincia cinese di Fujian. Zayton era la città portuale più prospera all'epoca di Marco Polo, e lui stesso è partito da qui per rientrare in patria alla fine del tredicesimo secolo ${ }^{19}$. Lì tuttora si conservano diverse lapidi, con una iscrizione di varie lingue, prodotte durante la dinastia Yuan, cioè l'impero sino-mongolo, che è durato dal $1279 \mathrm{al} 1368^{20}$. La lapide più importante è quella di Andrea da Perugia, vescovo francescano della città morto qui nel 1332 (fig.4). E' conosciuto per la lettera datata del 1326, che testimonia la presenza di conventi francescani a Zayton, uno dei quali poteva ospitare ventidue frati ${ }^{21}$. L'iscrizione della lapide non è sufficientemente leggibile, e perciò l'attribuzione di essa al frate-vescovo è stata messa in dubbio ${ }^{22}$. Inoltre, l'iscrizione è troppo piccola rispetto alla superficie (c’è un margine troppo modellati in stile tutto italiano.

\footnotetext{
${ }^{14}$ Per la bibliografia aggiornata sulla missione francescana in Asia, rimando a F. E. REICHERT, Incontri con la Cina. La scoperta dell'Asia Orientale nel medioevo, trad. A. SBERVEGLIERI, Milano, 1997; A. CACCIOTTI, M. MELLI (a cura di), I francescani e la Cina. Un'opera di oltre sette secoli, Milano, 2013 (soprattutto i seguenti saggi: A. MELLONI, "Mendicanti sulle vie dell'Estremo Oriente, 1245-1370. Diplomazie e missioni pontificie fra Innocenzo IV e Urbano V," pp. 21-45; R. SACCENTI, "La bibbia alla corte del Khan: La 'bibbia di Marco Polo," pp. 47-71; P. SELLA, "I mirabilia mundi nei viaggi e nelle permanenze dei primi frati minori in Cina (secoli XIII-XIV)," pp. 91-147.).

${ }^{15}$ Odorico da Pordenone (B. Odoricus de Portu Naonis), Relatio, cap. VIII, in: Sinica Franciscana, I, Quaracchi, 1929, pp. 424-439.

${ }^{16}$ M. SEIDEL, "Wiedergefundene Fragmente eines Hauptwerks von Ambrogio Lorenzetti," Pantheon, 36, 1978, pp. 119-127; Idem, "Gli affreschi di Ambrogio Lorenzetti nel Chiostro di San Francesco a Siena," Prospettiva, 18, 1979, pp. 10-20. Nel famoso affresco di Ambrogio Lorenzetti, eseguito per la sala capitolare dello stesso convento di Siena e trasportato ora nell'attigua chiesa, sono incluse figure centro-asiatiche con motivi esotici come un copricapo a forma di cono. Per alcuni studiosi questo dipinto descrive l'episodio di Tana. Comunque sia, le scene del Martirio dei francescani rappresentano, insieme alla Prova del Fuoco, una novità iconografica dovuta ad una esperienza nuova dei francescani. Cfr. H. YAKOU, "Memory without Mementos: Franciscan Missions and Ambrogio Lorenzetti's Frescoes in Siena," The Annual Report on Cultural Science (Hokkaido University), 119, 2006, pp. 1-17.

${ }^{17} \mathrm{La}$ scena corrisponde alla descrizione dei Commentarii di Ghiberti, “...... e paluesi, essere la grandine folta in su e paluesi, pare ueramente che'lla grandine balçi in su paluesi con uenti marauiglosi”. J. VON SCHLOSSER, Lorenzo Ghibertis Denkwürdigkeiten (I Commentarii), Berlino, 1912, p. 41; M. SEIDEL, “Gli affreschi di Ambrogio Lorenzetti... op. cit. (n. 16), p. 18.

${ }^{18}$ Odorico da Pordenone, op. cit. (n. 15), pp. 432-433. Cfr. anche A. ANDREOSE (a cura di), Libro delle nuove e strane e meravigliose cose. Volgarizzamento italiano del secolo XIV dell'Itinerarium di Odorico da Pordenone, Padova, 2000, pp. 146-152 (Itinerarium, capp. XIII-XVI), p. 150. La didascalia per quest'opera di Ambrogio nel L'arte di Francesco... op. cit. (n. 10), p. 447, fig. 1, specifica che essa raffigura la città di Amaliq (sic), ma il testo scritto da P. ARANHA non da spiegazione.

${ }_{19}$ Odorico da Pordenone, op. cit. (n. 15), pp. 46o-461; Marco Polo, Il Milione, cap. 157. Seguo la numerazione dei capitoli di The Description of the World, ed. A. C. MOULE, P. PELLIOT, 2 voll., London, 1938.

${ }^{20}$ W. WU, Religious Stone Inscriptions at Quanzhou, a cura di Y. WU, nuova ed. rev. e ampl., Beijing, 2005.

${ }^{21}$ Ibidem, pp. 373-376; Andrea da Perugia (Fr. Andreas de Perusia), Epistola, in Sinica... op.cit. (n. 15), pp. 373-377.

${ }^{22}$ H. FRANKE, "Sino-Western Contacts under the Mongol Empire," Journal of the Hong Kong Branch of the Royal Asiatic Society, 6, 1966, pp.49-72, 57.
} 


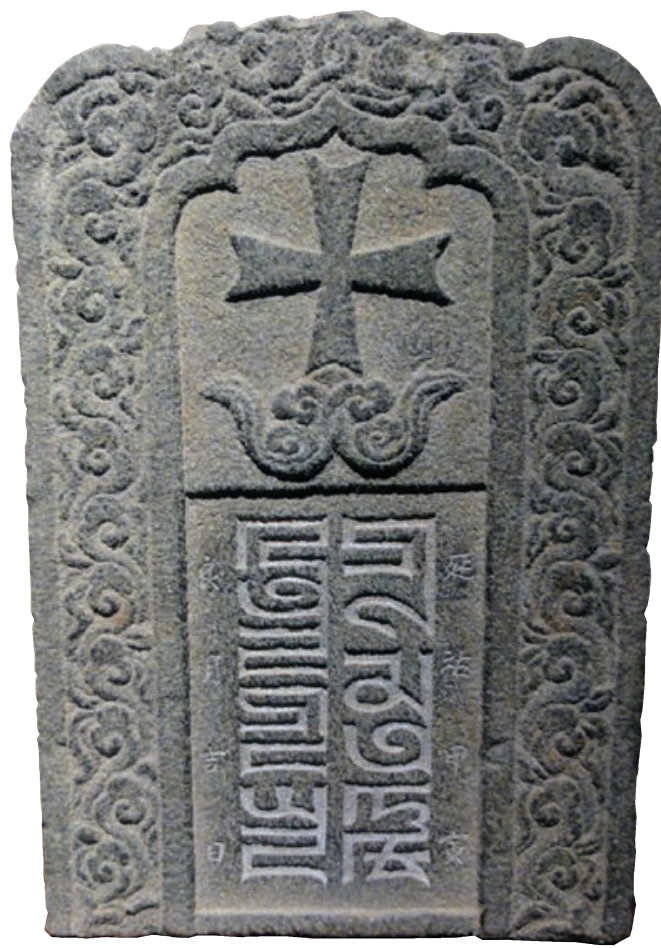

Fig. 5. Lastra tombale di sig. ra Yang, moglie di sig. Ye, Quanzhou, Museo marittimo.

ampio) e ai rilievi di due angeli sovrastanti, tanto da far dubitare dell'autenticità della lapide $^{23}$.

Più suggestiva è la lapide dedicata alla signora Yang, moglie del sig. Ye, recante un'iscrizione in due righe nell'alfabeto di Phags-pa, accompagnato dai caratteri cinesi $^{24}$ (fig. 5). L'alfabeto di Phags-pa è la scrittura ufficiale dell'impero mongolo, inventata su ordine dell'imperatore Khubilai ${ }^{25}$. Sopra questa scritta si trova una croce appoggiata su nuvole. Sono nuvole propizie, simbolo del pensiero taoista. L'introduzione dei motivi di religioni diverse dal cristianesimo è una caratteristica comune delle croci di Zayton. Si possono trovare anche deità volanti assimilabili agli angeli e fiori di loto. Questi ultimi sono un simbolo buddista del cielo ad ovest, cioè il paradiso, che si troverebbe nell'estremità ovest del mondo, come si vede in una lapide datata al $1289^{26}$ (fig. 6).

A Quanzhou, le steli tombali sono state trovate in quantità considerevole, ma solo con simboli semplici. Se vogliamo spostarci un po' a nord, nella città di Yangzhou in provincia di Jiangsu si trovano due lapidi con immagini più complesse (figg. 7, 8). Sono le uniche che finora siano state scavate fuori dalla città della provincia di Fujian, e confermano la presenza

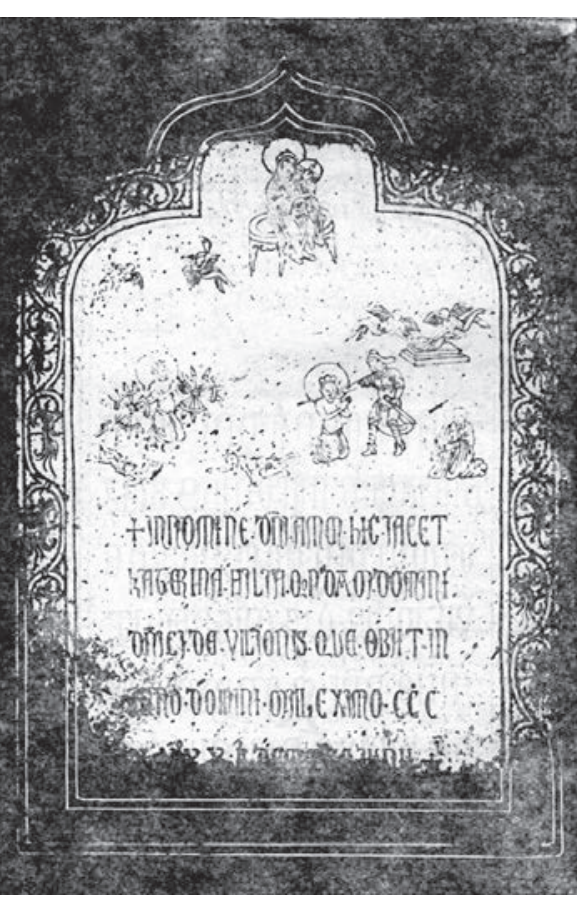

Fig. 7. Lastra tombale di Katerina Vilioni † 1342), Yangzhou (da N. Xia, 1979, p. 533, fig. 1).

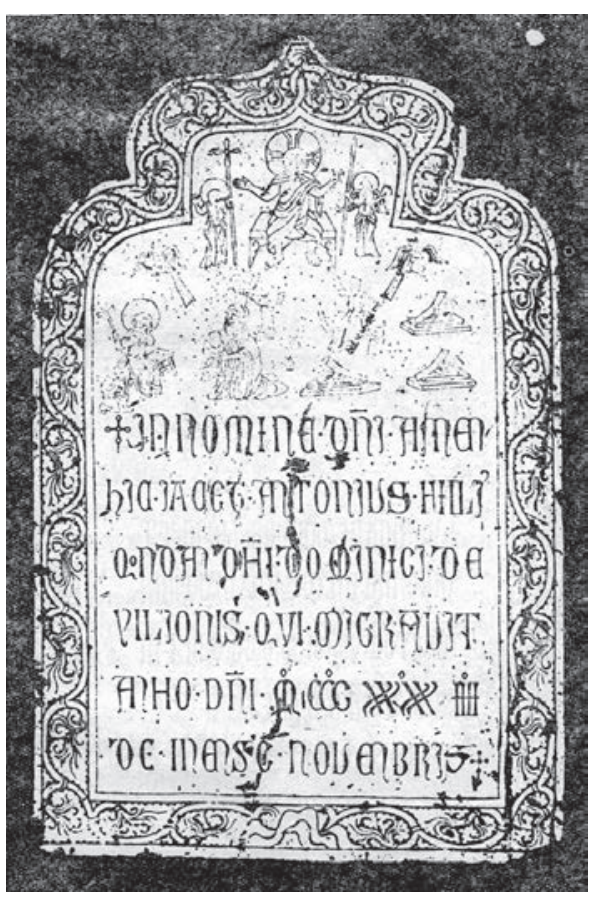

Fig. 8. Lastra tombale di Antonio Vilioni († 1344), Yangzhou, (da N. Xia, 1979, p. 534, fig. 2).

di una colonia di mercanti italiani. Odorico da Pordenone testimonia che esisteva un convento francescano a Yangzhou e Marco Polo insiste di avervi governato per tre anni ${ }^{27}$. Le lapidi sono di una famiglia genovese, i Vilioni. Una è dedicata a Katerina Vilioni, morta nel 1342, e l'altra al fratello Antonio, morto nel 1344. Sono state studiate da archeologi cinesi e recentemente anche da studiose americane, Lauren Arnold and Jennifer Purtle ${ }^{28}$.

L'iconografia è coerente nella lastra di Caterina, dove si riconoscono tre scene principali tratte dalla vita di Santa Caterina d'Alessandria: il supplizio con le ruote, la decapitazione, e il trasporto del corpo della santa a monte Sinai. In cima siede la Madonna col Bambino. Invece, sulla tomba di Antonio, si vede il santo onomastico, eremita con il bastone a

\footnotetext{
${ }_{23}$ Gli angeli sostengono il piedistallo, probabilmente a forma di loto, di una croce, la cui parte di sopra è troncata e dispersa. J. Foster, "Crosses from the Walls of Zaitun," The Journal of the Royal Asiatic Society of Great Britain and Ireland, s. n., 1954, pp. 1-25, 17. Si vedano anche le voci scritte da B. FELCI, P. SELLA, M. NICOLINI-ZANI, N. CELLI, nel L'arte di Francesco... op. cit. (n. 10), pp. 449, 452-454. Una possibile spiegazione per la lavorazione mediocre è che le lastre con angeli portacroce o con altri soggetti siano una specie di prêt-à-porter, dove basta inserire un'iscrizione per l'eventuale acquirente.

${ }^{24}$ È stata scavata nel villaggio di Houmao a Quanzhou nel 1985. W. WU, op. cit. (n. 20), pp. 409-411.

${ }^{25}$ Sulla scrittura di Phags-pa, introdotta come motivo ornamentale nell'arte italiana dell'epoca, cfr. H. TANAKA, “Oriental Scripts in the Paintings of Giotto's Period," Gazette des Beaux-Arts, 113, 1989, pp. 214-226.

${ }^{26}$ W. WU, op. cit. (n. 20), pp. 380-381.

${ }^{27}$ Odorico da Pordenone, op. cit. (n. 15), pp. 469-471; Marco Polo, Il Milione (n. 18), cap. 144.

${ }_{28}^{28}$ J. PURTLE, “The Far Side: Expatriate Medieval Art and Its Languages in Sino-Mongol China," Medieval Encounters, 17, 2011, pp. 167-197, 177-184; L. ARNOLD, Princely Gifts and Papal Treasures. Franciscan Mission to China and its Influence on the Art of the West, 1250-1350, San Francisco, 1999, pp. 134-147; N. Xia, "Latin Tombstones of Yangzhou and the Venetian Silver Coins from Guanzhou," Kaogu (Archeology), 1979, pp. 532-537.
} 


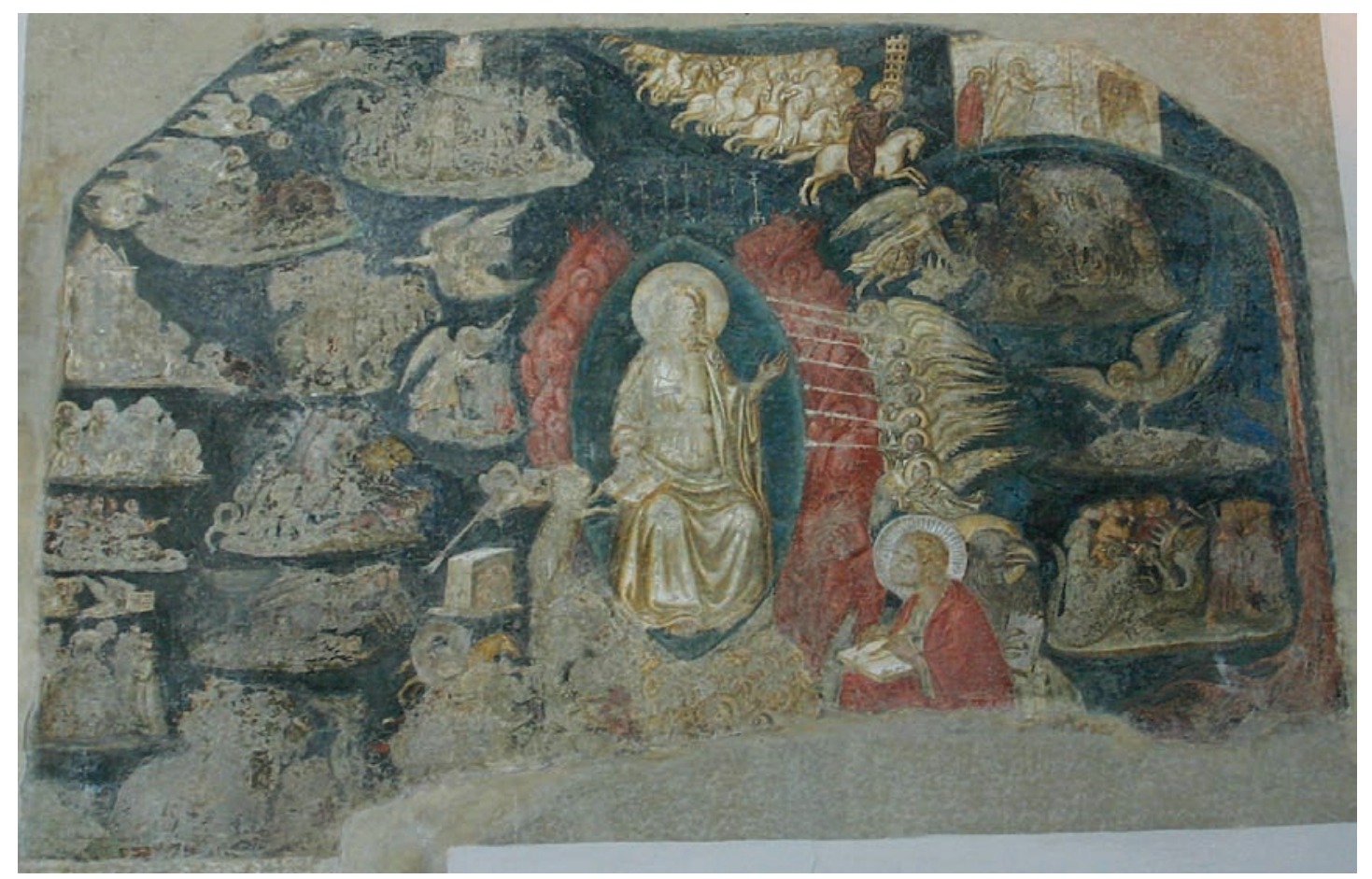

Fig. 9. Pietro Cavallini (?), Le scene dell'apocalisse, Napoli, la Chiesa di Santa Maria Donnaregina.

forma di Tau, ma davanti a lui c'è immagine della Madonna. Si è fatta quindi confusione con Sant'Antonio di Padova. Al lato opposto è collocata la resurrezione della carne alla fine del mondo, mentre in alto è raffigurato il Cristo giudice accompagnato da due angeli con i simboli della Passione ${ }^{29}$.

Questa immagine, insieme alla calligrafia latina, può essere definita puramente occidentale, ma la composizione delle varie scene sembra un po' particolare. Anche Jennifer Purtle, che ha analizzato brevemente queste lapidi, indica che il modo di collocare i diversi episodi, senza riquadri o linee dell'orizzonte, pare un po' anomalo, e lo collega con dipinti buddisti dell'epoca Yuan ${ }^{30}$. Questa modalità di composizione mi ricorda vagamente un riquadro che raffigura le diverse visioni dell'Apocalisse, ma tutte insieme e non in sequenza cronologica. Un esempio si può trovare sopra l'ingresso della Cappella Loffredo nella chiesa di Donnaregina a Napoli (fig. 9) $)^{31}$.

Un altro esempio di composizione difficile da spiegare nel solo contesto europeo è un famoso paesaggio del contado di Siena dipinto da Ambrogio Lorenzetti nella Sala dei Nove, raffigurato a volo d'uccello. Per questo dipinto è già stata avanzata l'ipotesi audace di un'influenza dell'arte pittorica cinese $^{32}$. Senza lasciar correre troppo la fantasia, vorrei far notare che il paesaggio è stato dipinto da tre punti di vista diversi allo stesso tempo: non è presente solo il punto di vista a volo di uccello, ma alcune case sono viste dal basso verso l'alto, per dare un'impressione di maestà ed imponenza. Questo modo di vedere - da più punti di vista, dall'alto, dal livello del suolo, e dal basso al tempo stesso - è molto comune nella pittura cinese soprattutto a partire dall'un- dicesimo secolo, nel periodo della dinastia Song, quando si è vista la fioritura del paesaggio dipinto in bianco e nero con inchiostro.

Tornando a Zayton, vorrei notare che in questa città e nelle zone limitrofe la produzione di ceramiche è stata importante per un lungo periodo, dall'epoca antica fino al diciannovesimo secolo. Si conoscono le fornaci storiche, come quelle di Jinjiang Cizao yao, Anxi-yao e altre, e restano molti cimeli conservati nel museo civico di Guangzhou ${ }^{33}$. Le forme delle ceramiche viste nelle pitture italiane del Due e Trecento non sono molto chiare e quindi è difficile dire se sono arrivate dalla Cina o altrove, ma l'ipotesi proposta da uno studioso giapponese, Yukihiro Nomura, che l'iconografia degli angeli che dedicano i fiori in vaso sia stata ispirata da una iconografia simile delle statue buddiste, andrebbe tenuta in considerazione quando vengono cercate le tracce di interscambi tra Oriente e Occidente ${ }^{34}$.

Come abbiamo visto, sono scarse le testimonianze dalle regioni dove erano attivi i missionari francescani, ed è quindi difficile dimostrare con prove dirette quanto davvero sia stata significativa l'influenza reciproca di stile e di iconografia nella produzione artistica del Basso Medioevo. Di certo, l'esperienza concreta del mare e l'espansione commericale non hanno contribuito molto alla nascita di una nuova iconografia dei paesaggi marini. Per il momento, possiamo concludere che l'impatto che lo scambio tra mondi diversi ha avuto sulla espressività di immagini, come quelle create da Ambrogio Lorenzetti, può essere spiegato dal clima nuovo dell'epoca in cui si è consolidato il rapporto diretto tra Occidente e Oriente.

${ }_{29}$ J. PURTLE, op. cit. (n. 28), p. 180; L. ARNOLD, op. cit. (n. 28), p. 139.

${ }^{30}$ J. PURTLE, op. cit. (n. 28), pp. 182-184.

${ }^{31}$ Per la bibliografia sugli affreschi di Donnaregina, vedi P. LEONE DE CASTRIS, Pietro Cavallini. Napoli prima di Giotto, Napoli, 2013.

${ }^{32}$ H. TANAKA, "Fourteenth century Sienese painting and Mongolian and Chinese influences: The analysis of Simone Martini's works and Ambrogio Lorenzetti's major works," Bijutsushigaku (Art History), 7, 1985, pp.1-55.

33 Cizao Kiln. Archeological Research and Excavation Report of Jinjiang Cizao Kiln, Fujian, Fujian Museum, Jinjiang Museum (eds.), Beijing, 2011.

34 Y. NOMURA, “La 'Madonna di Ognissanti' di Giotto riconsiderata," Bijutsushigaku (Art History), 8, 1986, pp. 89-129. 